\title{
Número de Ramsey multicolorido em Grafos Multipartidos
}

\author{
Juliana Sanches \\ Programa de Pós-graduação em Matemática Aplicada - UFRGS \\ 91509-900, Porto Alegre, RS \\ E-mail: juu.sanches@gmail.com \\ Emerson L. M. Carmelo \\ Departamento de Matemática - UEM \\ 87020-900, Maringá, PR \\ E-mail: elmcarmelo@uem.br
}

Resumo: O número de Ramsey multipartido (cardinalidade das classes) em bicolorações foi introduzido por Day, Goddard, Henning e Swart em 2001. Em 2004 Burger e van Vuuren sistematizaram e formalizaram problemas extremais associados à classe de grafos multipartidos. Neste trabalho estendemos esta variante do número de Ramsey para um número arbitrário de cores. Propriedades de crescimento, conexões com a teoria de Ramsey clássica, limitantes inferiores e superiores foram obtidos.

Palavras-chave: grafo multipartido, coloração de arestas, teoria de Ramsey

\section{Introdução}

Seja $K_{r}$ o grafo completo com $r$ vértices. Dados inteiros positivos $n_{1} \geq 2$ e $n_{2} \geq 2$ lembramos que o célebre número de Ramsey $r\left(n_{1}, n_{2}\right)$ denota o menor natural $r$ tal que toda bicoloração das arestas de $K_{r}$, usando as cores vermelho e azul, apresenta cópia vermelha de $K_{n_{1}}$ ou cópia azul de $K_{n_{2}}$. Determinar os números de Ramsey tem sido um grande desafio na teoria dos grafos. De fato, somente são conhecidos alguns valores exatos deste número: $r(2,2)=2, r(3,3)=6$, e $r(4,4)=18$, mas $r(5,5)$ ainda é um problema aberto. Desde o artigo de Ramsey em 1930, algumas contribuições tem se concentrado em determinar limitantes superiores e inferiores, por exemplo, $43 \leq r(5,5) \leq 49$. Tabelas atualizadas dos limitantes estão disponíveis em [11].

Muitos conceitos, variantes e extensões foram introduzidas com o intuito de ajudar no cálculo dos números de Ramsey: número de Ramsey bipartido, número de Ramsey generalizado, etc (veja [7]). Para a nossa proposta, mencionamos a seguinte extensão: o número de Ramsey multicolorido $r\left(n_{1}, \ldots, n_{k}\right)$ é o menor natural $r$ tal que toda $k$-coloração de arestas de $K_{r}$ apresenta pelo menos uma cópia monocromática de $K_{n_{i}}$ na cor $i$, para $1 \leq i \leq k$. Se $n_{i}=n$ para todo $i$, este número é denotado por $r(n ; k)$. Por exemplo: $r(3 ; 2)=r(3,3)=6$, o valor único conhecido de um multicolorido número Ramsey clássica é $r(3 ; 3)=r(3,3,3)=17$, e $51 \leq r(3 ; 4) \leq 62$, conforme [11].

Em particular, Day, Goddard, Henning e Swart [5] estudaram colorações de grafos com mais de duas classes. Eles introduziram o Número de Ramsey Multipartido (cardinalidade das classes). Por sua vez, Burger e van Vuuren [4] sistematizaram e formalizaram problemas extremais associados à classe de grafos multipartidos. Seja $K_{s \times c}$ o grafo completo multipartido balanceado com $s$ classes de cardinalidade $c$. Dados inteiros positivos $s, n_{1} \geq 2, p_{1}, n_{2} \geq 2$, e $p_{2}$ o número multipartido de Ramsey (cardinalidade de classes) $m_{s}\left(K_{n_{1} \times p_{1}}, K_{n_{2} \times p_{2}}\right)$ é o menor natural $c$ tal que toda bicoloração de arestas de $K_{s \times c}$, utilizando as cores vermelho e azul, apresenta uma cópia vermelha de $K_{n_{1} \times p_{1}}$ ou uma cópia azul de $K_{n_{2} \times p_{2}}$. Estes números podem ser considerados como uma extensão dos números de Ramsey clássicos. Observe que $r\left(n_{1}, n_{2}\right)=a$ implica 
$m_{a}\left(K_{n_{1} \times 1}, K_{n_{2} \times 1}\right)=1$. Note também que $m_{2}\left(K_{2 \times n_{1}}, K_{2 \times n_{2}}\right)=b\left(n_{1}, n_{2}\right)$, neste sentido este número de Ramsey generaliza o número Ramsey bipartido $b\left(n_{1}, n_{2}\right)$, onde este número denota o menor natural $b$ tal que toda bicoloração das arestas de $K_{2 \times b}$ apresenta cópia monocromática de $K_{2 \times n_{1}}$ na cor vermelha ou cópia azul de $K_{2 \times n_{2}}$ (vide [8]).

Vários resultados sobre os números de Ramsey em multipartidos são apresentados em [4], alguns deles em conexão com os números clássicos $r\left(n_{1}, n_{2}\right)$. Além disso, os limitantes gerais são obtidos, incluindo um limite inferior usando o método probabilístico.

Neste trabalho estendemos o número de Ramsey multipartido (cardinalidade das classes) para um número arbitrário de cores, como descrevemos na próxima seção. Discutimos as conexões com os números de Ramsey multicoloridos. Vários resultos em [4] foram estendidos, incluindo generalizações de limitantes superiores e inferiores.

\section{Definições e existência}

O grafo $K_{s \times c}$ denota o grafo mutipartido com $s$ classes de cardinalidade $c$

$$
K_{s \times c}=K_{c, c, \ldots, c}(s \text { times }) .
$$

Observe que $K_{n \times 1}=K_{n}$, mas $K_{1 \times n}$ denota o complementar de $K_{n}$ ( $n$ vértices isolados).

Dados números naturais $s, n_{i}, p_{i}$, com $1 \leq i \leq k$. O número de Ramsey multipartido multicolorido (cardinalidade das classes) $m_{s}\left(K_{n_{1} \times p_{1}}, \ldots, K_{n_{k} \times p_{k}}\right)$ é o menor natural $c$ tal que qualquer $k$-coloração arbitrária das arestas de $K_{s \times c}$ contém uma cópia monocromática de $K_{n_{i} \times p_{i}}$ na cor $i$, para algum $1 \leq i \leq k$.

No caso diagonal: $n_{i}=n$ e $p_{i}=p$ para todo $i$, com $1 \leq i \leq k$, é simplesmente denotado por $m_{s}\left(K_{n \times p} ; k\right)$. Neste trabalho consideraremos preferencialmente o caso diagonal.

Observemos que $r(n ; k)=a$ implica $m_{a}\left(K_{n \times 1} ; k\right)=1$. Ou seja, podemos dizer que o número de Ramsey multipartido multicolorido é uma extensão do número de Ramsey clássico multicolorido. Notemos também que $m_{2}\left(K_{2 \times n} ; k\right)=b(n ; k)$, em particular, este número de Ramsey generaliza o número de Ramsey bipartido multicolorido.

A existência deste número está condicionada a um valor da função de Ramsey clássica multicolorida. A versão para duas cores do resultado a seguir foi obtida por Burger e van Vuuren [4].

Teorema 1 Dados $n, k \geq 2$ e $p \geq 1$ números naturais arbitrários, o número de Ramsey multipartido multicolorido $m_{s}\left(K_{n \times p} ; k\right)$ existe se, e somente se $s \geq r(n ; k)$.

\subsection{Explicando a existência}

Para dar a ideia da demonstração do Teorema 1, e para relacionar o número multipartido com o número clássico de Ramsey, defini-se um novo conceito: a chamada coloração expansiva, descrita abaixo.

Definição 2 Uma coloração de arestas de $K_{s \times c}$ é chamada coloração expansiva se, para cada par de classes de $K_{s \times c}$, as arestas entre todos os vértices destas duas classes têm a mesma cor.

Um fato crucial segue: toda coloração expansiva de $K_{s \times c}$ corresponde a uma coloração de arestas de $K_{s}$ (isto pode ser feito identificando cada classe de $K_{s \times c}$ por um único vértice). Neste caso, dizemos que uma coloração expansiva de $K_{s \times c}$ é induzida por uma coloração correspondente de $K_{s}$. 

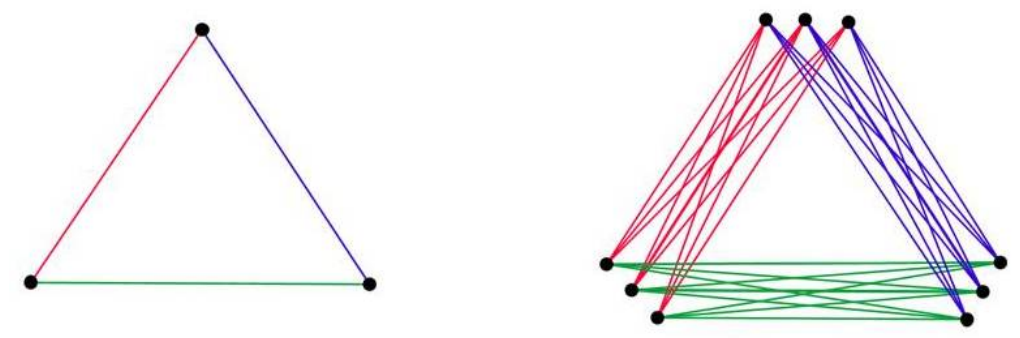

Figura 1: Coloração expansiva de $K_{3 \times 3}$ induzida por uma coloração de $K_{3}$

Esboço da demonstração do Teorema 1: Queremos mostrar que $m_{s}\left(K_{n \times p} ; k\right)$ existe, se e somente, $s \geq r(n ; k)$. Seja $r=r(n ; k)$. Primeiramente, suponhamos que $s \geq r(n ; k)$. A ideia da demonstração é garantir a existência de um $c$ "suficientemente grande"tal que qualquer $k$ coloração de $K_{r \times c}$ contém um subgrafo $K_{r \times d}$ cuja coloração é expansiva e $d \geq p$. Dessa forma pela definição de $r$ existe um $K_{n \times d}$ monocromático e como $K_{n \times p} \subseteq K_{n \times d}$, segue que $K_{r \times c}$ contém cópia monocromática de $K_{n \times p}$. Para provar a recíproca, provamos que $m_{s}\left(K_{n \times p} ; k\right)$ não existe se $s<r(n ; k)$. Como $r(n ; k)>s$, existe uma $k$-coloração de $K_{s}$ desprovida de cópia monocromática de $K_{n}$. Assim a coloração expansiva de $K_{s \times c}$ induzida por essa coloração de $K_{s}$ não contém $K_{n}$ monocromático. Consequentemente, $K_{s \times c}$ com essa coloração não contém $K_{n \times p}$ monocromático.

\section{Relações e propriedades de crescimento}

A fim de prosseguirmos com a análise do número de Ramsey multipartido, precisamos calcular a cardinalidade mínima de cada classe de $G=K_{s \times c}$ para que $G$ contenha obrigatoriamente um $K_{n \times p}$ como subgrafo. Afirmamos que este número é $\lceil p /\lfloor s / n\rfloor\rceil$.

Com efeito, seja $c$ o menor natural satisfazendo $K_{n \times p} \subseteq K_{s \times c}$. Quaisquer dois vértices de mesma classe de $K_{s \times c}$ não podem pertencer a classes distintas de $K_{n \times p}$. Isto significa que para obtermos $K_{n \times p}$ como subgrafo de $K_{s \times c}$, devemos agrupar classes inteiras de $K_{s \times c}$ com o intuito de formarmos $n$ novas classes. Desse modo, cada grupo deve conter $\lfloor s / n\rfloor$ classes de $K_{s \times c}$. Para assegurarmos que existem $p$ vértices em cada grupo, cada classe de $K_{s \times c}$ deverá conter pelo menos $\lceil p /\lfloor s / n\rfloor\rceil$ vértices.

Exemplo 3 Para $s \geq n \geq 2, p \geq 1$ arbitrários,

$m_{s}\left(K_{2 \times 1}, \ldots, K_{2 \times 1}, K_{n \times p}\right)=\lceil p /\lfloor s / n\rfloor\rceil$.

De fato, consideremos $K_{s \times\lceil p /\lfloor s / n\rfloor\rceil}=G$ com uma k-coloração arbitrária. Se esta coloração contém alguma aresta na cor $i^{*}$, para alguma cor $i^{*}$ com $1 \leq i^{*} \leq k-1$, o grafo $G$ obrigatoriamente contém um $K_{2 \times 1}$ monocromático. Caso contrário, todas as arestas de $G$ são da cor $k$, consequentemente, $G$ apresenta cópia monocromática de $K_{n \times p}$. E mais, $m_{s}\left(K_{2 \times 1}, \ldots, K_{2 \times 1}, K_{n \times p}\right)>\lceil p /\lfloor s / n\rfloor\rceil-1$. Coloridas as arestas de $K_{s \times(\lceil p /\lfloor s / n\rfloor\rceil-1)}=H$ todas com a cor $k$, o grafo $H$ não contém $K_{2 \times 1}$ na cor $i^{*}$ com $1 \leq i^{*} \leq k-1$, e nãa contém $K_{n \times p}$ monocromático.

(2) $m_{s}\left(K_{n \times 1} ; k\right)=1$, com $s \geq r(n ; k)$.

Com efeito, seja $r=r(n ; k)$ e suponhamos $s \geq r$. Consideremos uma $k$-coloração arbitrária de $K_{s \times 1}$. Como $K_{r \times 1} \subseteq K_{s \times 1}$, esta coloração induz uma $k$-coloração de $K_{r \times 1} \approx$ $K_{r}$. Pela definição de $r, K_{r}$ apresenta cópia de $K_{n}$ para alguma cor $i^{*}$. Consequentemente, o grafo $K_{s \times 1}$ apresenta cópia de $K_{n}$.

O próximo resultado apresenta uma propriedade de crescimento, que é uma generalização de um resultado em [4] 
Proposição 4 Dados números naturais $u, n, k \geq 2$ e s, $v, p$. Valem:

(1) $m_{s}\left(K_{n \times p} ; k\right) \leq m_{s}\left(K_{u \times v} ; k\right)$ sempre que $n \leq u$ e $p \leq v$ (quando ambos os números existem).

(2) $m_{s}\left(K_{n \times p} ; k\right) \leq m_{q}\left(K_{n \times p} ; k\right)$ se $q \leq s$ (quando ambos os números existem).

Prova: (1) Suponhamos a existência de $c=m_{s}\left(K_{u \times v} ; k\right)$. Seja uma $k$-coloração arbitrária de $K_{s \times c}$. Por hipótese, existe uma cópia monocromática de $K_{u \times v}$ para alguma cor $i$. Como $K_{n \times p} \subseteq K_{u \times v}$ o grafo $K_{s \times c}$ contém uma cópia monocromática de $K_{n \times p}$.

(2) Seja $c=m_{q}\left(K_{n \times p} ; k\right)$ um número finito. Consideremos $K_{s \times c}$ com uma $k$-coloração arbitrária. Como $q \leq s$, obrigatoriamente $K_{q \times c} \subseteq K_{s \times c}$. Assim, esta coloração induz uma $k$-coloração de $K_{q \times c}$. Pela definição de $c$, o grafo $K_{q \times c}$ contém uma cópia monocromática de $K_{n \times p}$ para alguma cor $i$. Consequentemente, $K_{s \times c}$ também a contém.

A proposição anterior permite que apresentemos um limite assintótico para o número de Ramsey multipartido. Um resultado analógo para duas cores é demonstrado por Burger e van Vuuren [4].

Teorema 5 Para $n \geq 2$ e $p, k \geq 1$ arbitrários,

$$
\lim _{s \rightarrow \infty} m_{s}\left(K_{n \times p} ; k\right)=1 .
$$

Prova: Seja $n_{0}=r(n ; k)$. A Proposição 4 e o Teorema 1 afirmam que a sequência

$$
\left\{m_{s}\left(K_{n \times p} ; k\right)\right\}_{s=n_{0}}^{\infty}
$$

é não-crescente. Como $m_{s}\left(K_{n \times p} ; k\right)$ é um número positivo para $s \geq n_{0}$, precisamos mostrar que $m_{q}\left(K_{n \times p} ; k\right)=1$ para algum número natural $q$.

Consideremos $q=r(n p ; k)$. O grafo $K_{q \times 1}$ é isomorfo a $K_{q}$. Por construção de $q$, toda $k$-coloração de $K_{q \times 1}$ contém cópia monocromática de $K_{n p}$. Mas, $K_{n \times p} \subseteq K_{n p}$ e portanto, $m_{q}\left(K_{n \times p} ; k\right)=1$.

O resultado a seguir fornece um limitante inferior, cuja demonstração é bastante simples, sendo este então um limitante inferior trivial. Este limitante possui versão para duas cores devido a Burger e van Vuuren [4].

Proposição 6 Sejam números naturais $s, p \geq 1$ e $n, k \geq 2$. Sob estas hipóteses,

$$
m_{s}\left(K_{n \times p} ; k\right) \geq\left\lceil\frac{n p}{s}\right\rceil \text {. }
$$

Prova: O grafo $K_{n \times p}$ possui $n p$ vértices. Dessa forma, devem existir pelo menos $\lceil n p / s\rceil$ vértices por classe de um grafo multipartido completo balanceado composto por $s$ classes a fim de garantir algum $K_{n \times p}$ como subgrafo.

\section{Limitante inferior via método probabilístico}

Em 1947 Erdős, por meio de argumentos probabilísticos, garantiu a existência de um $K_{t}$ monocromático em qualquer bicoloração do grafo completo com $2^{t / 2}$ vértices. Este método, atualmente conhecido por Método Probabilístico, é uma poderosa ferramenta utilizada para solucionar diversos problemas em matemática discreta. Por meio desta ferramenta, Burger e van Vuuren obtiveram um limitante inferior geral. Inspirados em tal resultado, no caso de $k$ colorações de um grafo multipartido apresentamos um limitante inferior geral. Este método não é construtivo, afirma a existência de um grafo multipartido "grande" desprovido de cópias monocromáticas de $K_{n \times p}$. 
Teorema 7 Sejam números naturais $s, p \geq 1$ e $n, k \geq 2$,

$$
m_{s}\left(K_{n \times p} ; k\right)>\frac{1}{j}\left(n !(p !)^{n} k^{p^{2}\left(\begin{array}{l}
n \\
2
\end{array}\right)-1}\right)^{\frac{1}{n p}}
$$

\section{Limitante superior via PCP}

O Princípio da Casa dos Pombos nos diz que: "Colocados $2 s-1$ pombos em duas casas, então a primeira tem pelo menos $s$ pombos ou a segunda tem pelo menos $s$ pombos". Reformulando esse enunciado, obtemos um enunciado equivalente usando a linguagem das colorações: "Para qualquer 2-coloração de $\{1, \ldots, 2 s-1\}$ existe um subconjunto monocromático com $s$ elementos na primeira cor ou um subconjunto monocromático com $s$ elementos na segunda cor". O PCP é o caso mais simples das partições do tipo Ramsey.

Neste ambiente, o PCP continua a ser uma ferramenta fundamental na demonstração de um limitante superior. Por meio do PCP generalizado, obtemos o seguinte limitante.

Teorema 8 Sejam números naturais $n \geq 1$ e $j, k \geq 2$, então

$$
m_{j}\left(K_{2 \times n} ; k\right) \leq \max \left\{k(n-1)+1,\left\lceil\frac{k(n-1)\left(\begin{array}{c}
k(n-1)+1 \\
n
\end{array}\right)+1}{j-1}\right\rceil\right\} .
$$

Os resultados anteriores estabelecem limitantes para o número de Ramsey multipartido. Por exemplo, pela Proposição $6, m_{4}\left(K_{2 \times 3} ; 3\right) \geq 2$ e o pelo Teorema $7, m_{4}\left(K_{2 \times 3} ; 3\right) \geq 3$. O teorema anterior garante que $m_{4}\left(K_{2 \times 3} ; 3\right) \leq 70$. Notemos que os resultados não fornecem limitantes "justos" para o número $m_{4}\left(K_{2 \times 3} ; 3\right)$. Assim como na Teoria Clássica de Ramsey, aqui em grafos multipartidos a obtenção de valores exatos mostra-se bastante complexa.

\section{Referências}

[1] Beineke, L. W. e Wilson, R. J.,Selected topics in graph theory,Academic Press, 1978.

[2] Bollobás, B.,Extremal Graph Theory, New York: Academic Press, 1978.

[3] Bollobás, B.,Modern Graph Theory, Springer, 1998.

[4] Burger, A. P. e van Vuuren, J. H., Ramsey numbers in complete balanced multipartite graphs. Part II: Size numbers, Discrete Math. 283 (2004) 45-49.

[5] Day, D., Goddard, W., Henning, M. e Swart, H., Multipartite Ramsey numbers, Ars Combinatoria, 58 (2001) 23-31.

[6] Diestel, R.,Graph Theory, Springer-Verlag, 1997.

[7] Graham, R. L., Rothschild, B. L. e Spencer J. H.,Ramsey Theory, John Wiley and Sons, New York, 1990.

[8] Hattingh, J. H. e Henning, M. A.,Bipartite Ramsey Theory, Utilitas Mathematica 53 (1998) $217-230$.

[9] Jukna, S.,Extremal combinatorics: with applications in computer science, Springer, 2001.

[10] Netto Boaventura, P. O.,Grafos: teoria, modelos, algoritmos, Edgard Blücher, São Paulo, 2003.

[11] Radziszowski, S.P., Small Ramsey numbers, The Electronic J. Combin., dynamical survey DS1.13, (2013). 